\title{
Complexity Theory of Psychopathology
}

Merlijn Olthof ${ }^{1}$, Fred Hasselman ${ }^{1,2}$, Freek Oude Maatman ${ }^{3,4}$, Anna M.T. Bosman ${ }^{1,2}$ \& Anna Lichtwarck-Aschoff ${ }^{5}$

${ }^{1}$ Behavioural Science Institute, Radboud University, Nijmegen, The Netherlands

${ }^{2}$ School of Pedagogical and Educational Sciences, Radboud University, Nijmegen, The Netherlands

${ }^{3}$ Philosophy of Behavioural Sciences, Faculty of Social Science, Radboud University, Nijmegen, The Netherlands

${ }^{4}$ Faculty of Philosophy, University of Groningen, Groningen, The Netherlands

${ }^{5}$ Faculty of Behavioural and Social Sciences, University of Groningen, Groningen, The Netherlands

Note: this manuscript is the final author version that was accepted for publication at the Journal of Abnormal Psychology. This version has not been proof-edited and is not the copy of record. The final article is available, upon publication, at the Journal of Abnormal Psychology (note that in 2022 the journal name will change to Journal of Psychopathology and Clinical Science).

Corresponding author: Merlijn Olthof, Behavioural Science Institute, Radboud University, Montessorilaan 3,6525HR, Nijmegen, The Netherlands. Email: merlijn.olthof@ru.nl 


\title{
COMPLEXITY THEORY OF PSYCHOPATHOLOGY
}

\begin{abstract}
There is a renewed interest for complex adaptive system approaches that can account for the inherently complex and dynamic nature of psychopathology. Yet, a theory of psychopathology grounded in the principles of complex adaptive systems is lacking. Here, we present such a theory based on the notion of dynamic patterns: patterns that are formed over time. We propose that psychopathology can be understood as a dynamic pattern that emerges from self-organized interactions between interdependent biopsychosocial processes in a complex adaptive system comprising a person in its environment. Psychopathology is emergent in the sense that it refers to the person-environment system as a whole, and cannot be reduced to specific system parts. Psychopathology as a dynamic pattern is also selforganized, meaning that it arises solely from the interdependencies in the system: the interactions between countless biopsychosocial variables. All possible manifestations of psychopathology will correspond to a wide variety of dynamic patterns. Yet, we propose that the development of these patterns over time can be described by general principles of pattern formation in complex adaptive systems. A discussion of implications for classification, intervention and public health concludes the paper.
\end{abstract}

Keywords: psychopathology; transdiagnostic; complex adaptive systems; dynamic patterns; development

\section{General Scientific Summary}

We propose a transdiagnostic theory of psychopathology based on the principles of pattern formation in complex adaptive systems. We discuss implications for classification, intervention and public health. 


\section{COMPLEXITY THEORY OF PSYCHOPATHOLOGY}

Psychopathology is an inherently complex phenomenon characterized by strong heterogeneity within diagnostic categories (Allsopp et al., 2019), comorbidity (Kessler et al., 2005) and variation over time (Caspi et al., 2020). In contrast to predictions of specific reductionist theories (e.g., 'dopamine theory of psychosis'), evidence is now accumulating that psychopathology is strongly individualized (Wright \& Woods, 2020) and results from a complex interplay between interdependent biological, psychological, and sociocultural factors (Frisch, 2016). These two aspects of psychopathology are both clinically intuitive and largely agreed upon by researchers, but have had surprisingly little influence on scientific practice. Most psychopathology research centers around group-level comparisons on specific biological or psychological factors (van Os et al., 2019). Thus, there appears to be a misfit between our current understanding of psychopathology, as a complex and individualized phenomenon, and the way it is generally studied.

A potential resolution lies in a complex systems paradigm where theory and methods can facilitate a more fine-grained, individualized, multi-level, and dynamic understanding of psychopathology (Nelson et al., 2017). While concepts and ideas from complexity science are gaining popularity in psychopathology research, their application is not always in line with the basic tenets of complex systems, leading to conceptual and methodological problems (Haslbeck et al., 2019; Olthof, Hasselman, \& Lichtwarck-Aschoff, 2020).

In this paper, we aim to provide a complexity theory of psychopathology that may resolve these problems and help the field forward. Rather than importing concepts from complexity science into existing psychopathology theory, we seek to construct such a theory based on complex systems principles (Haken, 1983; Schoner \& Kelso, 1988). In doing so, we aim to develop a general theoretical framework that enriches, complements, and integrates diverse fields in psychopathology research such as developmental psychopathology (Cicchetti \& Cohen, 2006), symptom networks (Borsboom \& Cramer, 2013), personalized 


\section{COMPLEXITY THEORY OF PSYCHOPATHOLOGY}

psychopathology (Wright \& Woods, 2020), psychotherapy research (Mahoney, 1991) and enactive psychiatry (de Haan, 2020). Our theory implies a radical strategy shift in psychopathology research, which, we argue, will reveal general aspects of psychopathology and clinical change that are highly clinically relevant.

The structure of our paper is as follows. First, we provide a general introduction of pattern formation in complex adaptive systems. Next, we explain how pattern formation may underlie psychopathology and mental health in a person-environment system. Third, we discuss how person-environment systems can be studied in order to shed further light on psychopathology and clinical change. We introduce novel hypotheses and concisely review current evidence and formulate directions for further research. Last, we discuss clinical and public health implications.

\section{Introduction to complex adaptive systems}

A complex adaptive system is a composition of parts and wholes in which interdependencies between parts lead to the emergence of wholes (van Geert, 2019). All living systems are considered complex adaptive systems, but ecosystems, certain electromagnetic systems and organizations also are typical examples (Ladyman et al., 2013). Complex adaptive systems are often nested: wholes can form parts of even larger wholes. For example, a cell is nested in an organ, which is nested in an animal, which in turn is nested in an ecosystem - each of which can be considered a complex adaptive system in itself. The future state of a complex adaptive system is generated by its current internal state in interaction with its external environment, leading to structures in time, called dynamic patterns (Kelso, 1997).

Common to all complex adaptive systems is that the dynamic patterns they exhibit as a whole cannot be readily understood from the behavior of the numerous underlying system 


\section{COMPLEXITY THEORY OF PSYCHOPATHOLOGY}

parts (Ladyman et al., 2013). Consider locomotion as an example. Locomotion is a highly organized dynamic pattern at the level of the entire human body, which arises from complex interactions between a huge number of parts, including approximately $10^{2}$ joints, $10^{3}$ muscles, and $10^{14}$ cells (Turvey, 1990). These parts can be understood as variables, representing the degrees of freedom available to the system to generate behavior. Understanding locomotion thus requires solving a problem of coordination, that is, how the organism masters these many degrees of freedom to generate coordinated dynamic patterns (Bernstein, 1967). Although the number of variables involved in movement is tremendous, locomotion as a whole is coordinated and can be described by only few collective variables, such as the relative phase or the frequencies of oscillatory limb movements (Schöner et al., 1990). Collective variables 'summarize' all complex interactions underlying them, thereby allowing one to assess the overall state of the entire system at a glance.

The problem of coordination is not restricted to movement, but extends to all pattern formation in complex adaptive systems. Complexity scientists explain pattern formation without relying on a central control unit; a unit that instructs pattern formation much like a conductor instructs an orchestra (Ladyman et al., 2013). Central control units are problematic as they lead to a logical regress with regard to what instructs the control unit. A control unit also cannot explain novel patterns, because they necessarily have to pre-exist within the controller (Van Orden et al., 2011). An alternative proposal is that dynamic patterns emerge from the interactions among the system parts themselves: patterns are self-organized (Haken, 1992). This also implies that dynamic patterns are emergent phenomena in the sense that they are exhibited by the system as a whole rather than by the underlying parts (Anderson, 1972).

Self-organization and emergence are best illustrated by paradigmatic non-living complex systems. Consider ferromagnetization: how electrons (i.e., parts) in a material can generate a dynamic pattern in the form of a macroscopic magnetization. Under normal 


\section{COMPLEXITY THEORY OF PSYCHOPATHOLOGY}

temperatures, the magnetic moments of the electrons are independent of one another, meaning that they all point in different directions. When the temperature decreases to a critical point, the magnetic moments of the electrons become correlated due to influence of the magnetic moments of their neighbors and all start to align, leading to the emergence of macroscopic magnetization. Note that magnetization is an emergent phenomenon: it is a property of the system as a whole, which does not exist at the level of the isolated parts (Anderson, 1972). Also, note that magnetization arises solely from self-organizing interactions between electrons and is thus not instructed by any type of control unit. While it is well-understood how lowering the temperature drives the self-organized magnetization at a critical temperature, the temperature itself does not contain any information about the magnetic field.

This example illustrates how self-organization occurs under two conditions: 1) system parts must be interdependent, meaning that they are continuously influencing each other, and 2) stable dynamic patterns must be available which the system can settle into. These two conditions are also met for the locomotion example above, and for movement in general. Interdependence between bones, joints, and muscles is immediately apparent through the physical connections between them, which reduces the degrees of freedom of the movement system as a whole (Turvey, 2007). Also, various movements such as sitting, crawling, walking, running, and so on, can be understood as stable dynamic patterns that form through development and become available to the system to settle into under certain conditions (Schöner et al., 1990; Thelen \& Smith, 1994).

Self-organized pattern formation in complex adaptive systems occurs through phase transitions. Phase transitions are sudden, qualitative changes in the global behavior of a complex system: changes at the level of the whole. In mono-stable systems, like a ferromagnet, a phase transition marks the onset of an emergent pattern (i.e., the macroscopic magnetization) from a situation in which there was no emergent pattern at all. Living systems, 


\section{COMPLEXITY THEORY OF PSYCHOPATHOLOGY}

in contrast, are multi-stable, meaning that there are many different stable patterns available, like in movement (Kelso, 2012).

A well-studied example of phase transitions in movement is infant stepping behavior. Soon after birth, an infant, when held upright with the feet on the ground, will move legs and feet as if stepping. This behavioral pattern disappears after about two months and then reappears about four to six months later. The disappearance and reappearance of this pattern had been a mystery for long time, and had been hypothesized to play an important role in the development of bipedal locomotion (initially a reflex that returns after brain maturation which places it under voluntary control). Thelen and Ulrich (1991) found that there was actually a very simple reason for the sudden disappearance and reappearance of the stepping behavior: the ratio between leg weight and leg strength. Infants can only perform stepping behavior when they have enough leg strength relative to their leg weight. After two months of age infants gain a disproportionally amount of weight that affects the ratio negatively, after a couple of months the muscles restore this ratio again to the point at which they can lift their legs again.

Leg strength and leg weight therefore function as so-called control parameters in the transitions from appearance via disappearance to reappearance of stepping behavior (Thelen \& Ullrich, 1991). Control parameters summarize constraints (such as leg weight and leg strength) that together determine which dynamic patterns are possible in a specific system (Van Orden et al., 2011). Changes in control parameters therefore drive the phase transitions between different patterns, which occur at certain critical values where new dynamic patterns become available (such as the tipping point where the leg is strong enough to lift its weight).

\section{A complexity theory of psychopathology}




\section{COMPLEXITY THEORY OF PSYCHOPATHOLOGY}

Complex systems have not just spawned interest in physics and biodynamics, but also inspired research in developmental psychology (Bronfenbrenner, 1977; Thelen \& Smith, 1994), clinical psychology (Schiepek \& Tschacher, 1992), psychotherapy (Mahoney, 1991), developmental psychopathology (Granic, 2005), psycholinguistics (Wijnants et al., 2012) and neuroscience (Freeman, 1992). In the remainder of the paper, we integrate these lines of work with the principles of complex adaptive systems introduced above, in order to arrive at a complexity theory of psychopathology. What we intend to show is that psychopathology can be seen as a dynamic pattern that emerges from self-organized interactions between interdependent biopsychosocial processes in a complex adaptive system comprising a person in its environment.

\section{Interdependence}

Interdependence of biopsychosocial processes is the most fundamental assumption of our theory. Interdependence means that biopsychosocial processes interact, in the sense that they continuously influence each other over time. This idea should be uncontroversial: physiology, psychology and sociocultural changes all influence each other, even in ways that are hard to conceive (e.g., socio-cultural changes have affected bone density; Fausto-Sterling, 2020). Interdependence has a long history in psychopathology and is prominent in the biopsychosocial model (Engel, 1977), but also in more modern conceptualizations of psychopathology such as those presented by symptom networks (Borsboom, 2016) and mechanistic property clusters (Kendler et al., 2011). For example, several researchers have argued interdependencies between symptoms may create positive or negative feedback loops

such as: insomnia $\rightarrow$ fatigue $\rightarrow$ concentration problems $\rightarrow$ rumination $\rightarrow$ insomnia $\rightarrow \ldots$ and so on (Borsboom \& Cramer, 2013; Hayes et al., 2015; Schiepek, 2003) 


\section{COMPLEXITY THEORY OF PSYCHOPATHOLOGY}

In complex adaptive systems, interdependence implies inseparability: it is impossible to isolate unique contributions of system parts, as any given state of one process is necessarily dependent on the states of all other processes (Van Orden et al., 2011). As a metaphor for inseparability, consider psychopathology as a piece of fabric in which all intertwined threads represent biopsychosocial processes. Separating the threads to study them makes it impossible to recover how they were intertwined in the fabric ${ }^{1}$. Interdependence thus implies that psychopathology cannot be fully understood by separating it into 'smaller pieces' (Van Geert, 2019; Wallot \& Kelty-Stephen, 2017).

This contrasts with the assumption of independence, the idea that system parts interact in an additive manner and can thus be isolated (Van Orden et al., 2003). According to the independence view, psychopathology is like a puzzle that consists of many biopsychosocial pieces. Following this view, studies tend to focus on the unique contributions of specific variables (e.g., serotonin levels, cognitive biases) in explaining a specific form of psychopathology (Figure 1). Although research has revealed relevant factors associated with psychopathology, so far, no set of variables has been discovered that fully describe a distinct form of mental disorder (e.g., Hasselman, 2015; Wittenborn et al., 2016). The reason, we advocate, is that many variables are involved interdependently rather than independently of one another.

Whether behavior indeed emerges from interactions between interdependent parts has been addressed in empirical studies that examined variability over (extended) time (for a review, see Wijnants, 2014). Such studies seek to determine the randomness/rigidness of the values of a variable measured over time (i.e., a time series). A time series that reveals a stable, predictable pattern of values is rigid, whereas a time series that yields unpredictable, widely varied values is random white noise. Systems of independent parts produce white noise,

\footnotetext{
${ }^{1}$ This metaphor was presented by Paul van Geert at the Jean Piaget Society conference 2019.
} 


\section{COMPLEXITY THEORY OF PSYCHOPATHOLOGY}

whereas systems of interdependent parts behave more rigidly (Wallot \& Kelty-Stephen, 2017). Notably, systems that behave halfway between rigid and random regimes seem to be most flexible or healthy (Van Orden et al., 2011). The term that is used for this type of variability is pink noise, as opposed to random white noise and rigid brownian motion. Pink noise in a time series is in fact a mixture of stable and unstable patterns that allow the system to quickly adapt to a changing environment.

Studies on behavioral variability have found numerous examples of pink noise in a wide variety of measures such as EEG, heart rate, postural sway, reaction and self-ratings (Wijnants, 2014). Moreover, various studies found deviations of pink noise to be related to pathology. Goldberger and colleagues (2002) for example showed that a healthy heart is characterized by a heart rate pattern with pink noise variability. A heart that beats too regularly or too randomly signifies pathology. Deviations from pink noise patterns also appear to be related to transdiagnostic psychopathology: higher rigidity compared to control participants has been found in reaction time series of individuals diagnosed with ADHD (Gilden \& Hancock, 2007) and dyslexia (Wijnants et al., 2012), and in postural sway data for individuals diagnosed with psychosis (Kent et al., 2012) and bipolar disorder (Bolbecker et al., 2011). Furthermore, decreasing rigidity over time in self-ratings has been found to predict clinical improvement in psychotherapy (Fisher \& Newman, 2016).

The implications of interdependence for psychopathology research practices are enormous. It means that we cannot decompose psychopathology into smaller pieces, but that we have to study and treat it as a whole. The notion of complex adaptive systems thereby provides further support to the holistic work of practitioners, who tend to see their patients as 'wholes'. Also, understanding psychopathology as a whole highlights the value of qualitative research on lived experience of psychopathology (Colombetti, 2012). Complex adaptive systems, however, also allow for a quantitative study of psychopathology, on which our 


\section{COMPLEXITY THEORY OF PSYCHOPATHOLOGY}

theory is focused. Key to such an approach is that psychopathology can be studied as a dynamic pattern and that, because dynamic patterns are coordinated, it is possible to describe the dynamics of psychopathology based on a relatively small number of collective variables.

A

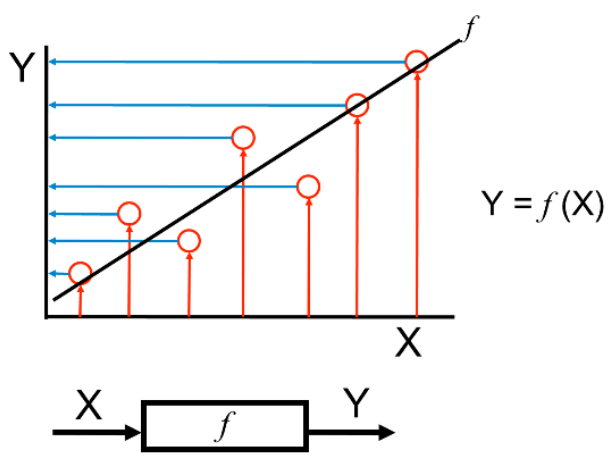

B

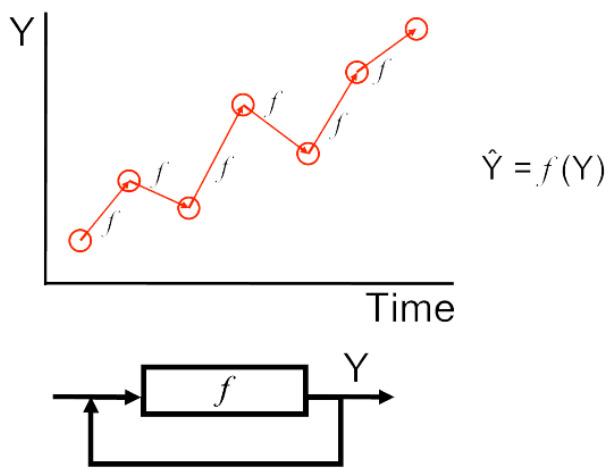

Figure 1. (A): Studying psychopathology throught the perspective of independence: Psychopathology (Y) explained as a function of another property (X). (B): Studying psychopathology throught the perspective of dynamic patterns: Psychopathology (Y) explained as a function of itself, a process over time. The current psychopathological state $(\mathrm{Y})$ generates its succesive state $(\hat{Y})$ through a mechanism or principle of change $(f)$.

\section{Dynamic patterns}

The basis of psychopathology as a dynamic pattern is that its structure emerges over time: it is a process, not a specific configuration of symptoms. Time has always played a central role in conceptualizing psychopathology. No-one would suggest that a momentary state of sadness is pathological, while a dynamic pattern of persistent sad mood often is. In this case, the dynamic pattern is a stable state, called an attractor: the system is pulled or attracted towards that specific behavior. An attractor forms a constraint on the degrees of freedom that are available to a system when generating its behavior; the system cannot 'move freely' but keeps being attracted to one dynamic pattern. For example, an individual keeps being attracted to a sad emotional state (i.e., a fixed-point attractor) or appears to be unable to escape a cycle of periodic shifts between sad and euphoric states (i.e., an oscillator or limit- 


\section{COMPLEXITY THEORY OF PSYCHOPATHOLOGY}

cycle attractor). In addition to fixed points and limit cycles, attractors can also yield more complex structures, such as quasi-periodic or even chaotic patterns (Strogatz, 2015). Which exact types of attractors are to be found in psychopathology is an open empirical question, but the important point is that the presence of attractors always signifies a specific constraint on the available degrees of freedom.

Attractors in psychopathology can be quantitatively studied as trajectories in state space, the space formed by the system's collective variables. The current state of the system is represented as a point in this space. The state space trajectory, then, is a historical record of the system's behavior, which provides insight in the presence of attractors. For example, we can identify a point attractor of sad emotion and a limit cycle between sadness and euphoria in a state space with a valence and an arousal dimension (see Figures $2 \mathrm{~A}$ and 2B). Due to interdependence, attractors of psychopathology will be reflected in various collective variables $^{2}$. For example, depression may be reflected as an attractor of the valence/arousal state space (e.g., persistent sad emotion), but also in state spaces that reflect cognition (e.g., rumination), overt behavior (e.g., inactivity), physiology (e.g., loss of appetite) and many possible other variables. It follows that if mood, cognition, overt behavior and physiology are considered collective variables that span the dimensions of a higher-level state space, depression would also be an attractor in this space. It is thus possible to take a collective variable out of the system, but not the system out of the variable (Van Geert, 2019).

Selecting different collective variables and measurement strategies can provide insights in psychopathology at different timescales (Eronen, 2019). Some dynamics are 'slow', such as an episode of depression or psychosis, which may be an attractor that is stable for months. Other dynamics are 'fast', such as a panic attack, which may be an attractor that

\footnotetext{
${ }^{2}$ This principle is formalized in Takens theorem, which yields that interdependent variables 'carve into' one another (e.g. the dynamics of mood are in the dynamics of cognition and vice versa), which makes it possible to derive the global dynamics of the system as a whole out of only one variable (Takens, 1981).
} 


\section{COMPLEXITY THEORY OF PSYCHOPATHOLOGY}

only exists for minutes. Specifically, the measurement length and sampling frequency determine the timescales at which attractors can be identified (Thelen \& Smith, 1994). Nevertheless, attractors on different timescales are not separate processes, but different aspects of the system as a whole. For example, panic disorder involves slow-evolving avoidance tendencies and beliefs about arousal, but also fast-evolving repeated panic attacks (Robinaugh et al., 2019); both can be viewed as attractors.

The notion of an attractor resonates with the proposal of understanding psychopathology as a 'stuck state': psychopathology is not attributed to the experience of certain symptoms per se, but to the impossibility to disengage from them (Holtzheimer \& Mayberg, 2011). For example, anxiety upon encountering a spider is in itself generally not seen as pathological. It is, however, evaluated as pathological when one cannot disengage from such anxiety and when anxiety persists even when spiders are not encountered for a long time. The notion of an attractor therefore supports the claim that psychopathology relates to rigidity (Bonnano et al., 2005; Hollenstein et al., 2013), as is also supported by the findings on reduced flexibility and deviations of pink noise as discussed above.

Not only psychopathological states can be seen as attractors: healthy mental states should also be considered attractor states. Mental health is also a stable pattern over time, although possibly a more flexible one than psychopathological alternatives. Mental health as an attractor relates to the concept of resilience; the ability to 'bounce back' in response to stressors (Masten, 2001). For example, it is considered healthy when a person experiences momentary anxiety in response to a spider, but quickly recovers to a non-anxious state. In complex systems terms, resilience is formally defined as resistance to change. An attractor implies resistance to change, as persons will return to their attractor state after a perturbation

(e.g., encountering a spider). Notably, for dynamic patterns associated with health, resistance 


\section{COMPLEXITY THEORY OF PSYCHOPATHOLOGY}

to change is desirable, whilst for dynamic patterns associated with psychopathology it is often not.

(A) Point Attractor

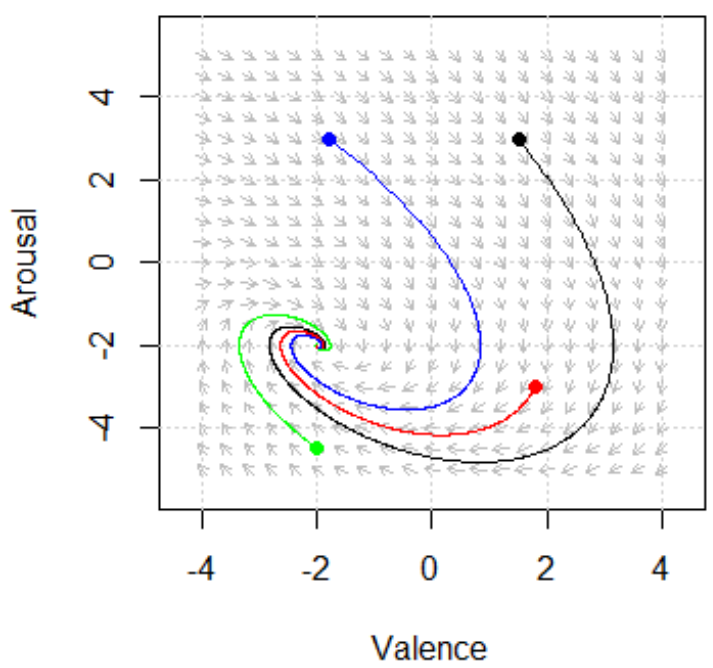

(B) Limit Cycle

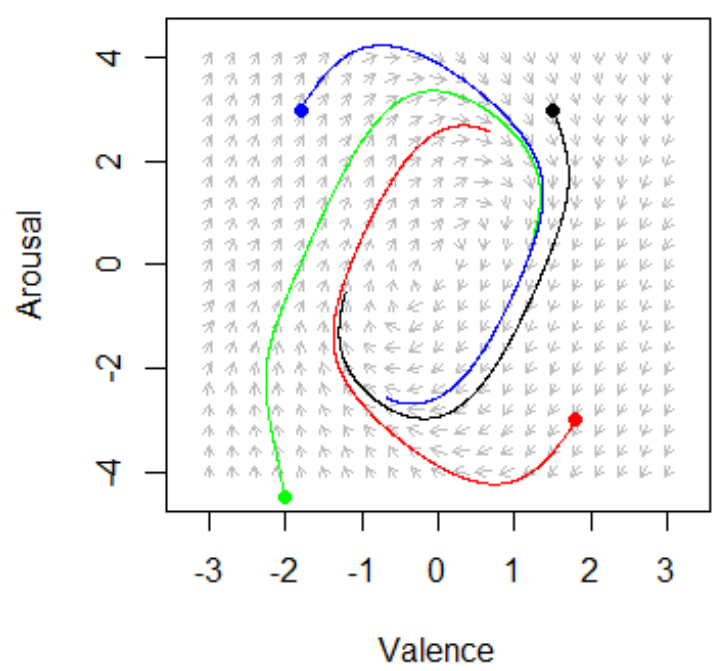

Figure 2. Simulated illustration of attractors in a two-dimensional valence/arousal state space. Starting at different points in state space, all possible system trajectories (indicated by different colors) are attracted to (A): a point attractor of a sad mood, (B): a limit cycle attractor between sad mood and euphoria.

\section{Phase transitions in psychopathology}

When the system trajectory moves from being dominated by one attractor to being dominated by another, a phase transition takes place (Schoner \& Kelso, 1988). Our theory therefore proposes that qualitative changes in psychopathology - such as onset, relapse and recovery - can be understood as phase transitions. Yet, we do not propose that all clinical changes are phase transitions. Often, change will consist of natural changes within one attractor state. Since the attractor in such a scenario keeps attracting the system, change will not be enduring. For example, a person who is stuck in a depressed state may experience short-lived moments of joy, but will quickly return to a sad mood state afterwards (such as visualized in Figure 3A). Phase transitions, in contrast, yield enduring changes because a person stabilizes in a completely new attractor (such as in Figure 3D). For example, a person 


\section{COMPLEXITY THEORY OF PSYCHOPATHOLOGY}

may transition from a period of depression to a period of mental wellbeing, which lasts far longer than a moment of joy. In contrast to within-attractor changes, phase transitions yield enduring change that is not easily reversible.

The proposal that enduring clinical change reflects phase transitions leads to several predictions. First, this hypothesis predicts that we should be able to observe qualitative changes in the dynamics of collective variables. Clinical change, including the onset of psychopathology, or the recovery from it, should thus be reflected as qualitative change in repeated measures of these variables. Often, such a qualitative change will be abrupt, but there can also be a gradual change from one attractor to another (Kéfi et al., 2013). While often not phrased in complex system terms, the phase transition hypothesis has been extensively studied in research on sudden gains: sudden improvements that occur in treatment. Sudden gains are generally defined as considerable improvements in symptom levels from one therapy session to another, lasting for at least three sessions (Tang \& DeRubeis, 1999). Studies have shown that sudden gains are a common phenomenon across patients with various diagnoses and in various treatment settings (for a meta-analysis, see Shalom \& Aderka, 2020), including pharmacological or placebo treatment (Vittengl et al., 2005). Moreover, sudden gains are related to better treatment outcomes in general and at follow-up assessments, and thus seem to represent enduring clinical change (Shalom \& Aderka, 2020). Studies have also found sudden losses in psychotherapy, which occur less frequently but also seem related to worse outcomes (Lutz et al., 2013), as one would predict from the phase transition hypothesis.

Sudden gains and losses have so far been difficult to explain. The original explanation - that cognitive changes induced by cognitive-behavioral therapy for depression lead to sudden gains (Tang \& DeRubeis, 1999) - does not fit findings that sudden gains also occur in patients with different diagnosis and different treatments. Also, more recent explanations are still primarily focused on sudden gains in therapy, often ignoring sudden gains in the absence 


\section{COMPLEXITY THEORY OF PSYCHOPATHOLOGY}

of therapy and sudden losses (e.g. Zilcha-mano et al., 2019; Aderka \& Shalom, 2021).The phase transition hypothesis may provide a more parsimonious explanation for sudden gains and losses. After all, recall that phase transitions are a general principle of change in complex adaptive systems and thus should describe qualitative change irrespective of patient, diagnosis, treatment characteristics, and direction of change (either sudden gains or losses). Furthermore, phase transitions constitute more enduring changes than gradual within-attractor changes, which explains the relation between sudden gains and better outcome.

Second, the phase transition hypothesis predicts that loss of stability plays an essential role in clinical change. In order for clinical change to occur, the existing attractor first needs to destabilize. Destabilization has a long history in psychotherapy research, where it is theorized as an important aspect of a system-wide reorganization (Hayes et al., 2007; Mahoney, 1991). In complexity science, destabilization is defined as a loss of attraction in the current attractor, which gives rise to particular dynamic phenomena called critical fluctuations and critical slowing down (e.g., Kelso et al., 1986). As the potential landscape changes from a deep to a shallow well during destabilization, the system gains more degrees of freedom, leading to more variable behavior: critical fluctuations ('the ball moves around more freely', Figure 3B). Similarly, the time it takes to return to the attractor after perturbation increases during destabilization: critical slowing down. Because these increases in fluctuations and return time precede the actual tipping point of the transition, they can be interpreted as early-warning signals for upcoming clinical transitions (Scheffer et al., 2009).

Destabilization has been extensively studied as a predictor of clinical change. Several studies on psychotherapeutic change found destabilization, measured as increased fluctuations, in both coded observational data of therapy sessions and repeated self-ratings to relate to better treatment outcome (reviewed in Hayes \& Andrews, 2020). Outside the therapeutic context, fluctuations in repeated self-ratings are also related to increases in 


\section{COMPLEXITY THEORY OF PSYCHOPATHOLOGY}

depressive symptoms for at-risk individuals and to decreases in depressive symptoms for currently depressed individuals (van de Leemput et al., 2014). A limitation of many studies is that they yield a between-person comparison of instability, which is not a direct test of the phase transition hypothesis that instability should rise over time within the person (Bos \& De Jonge, 2014). In a recent study, we found that increased fluctuations within the person can predict upcoming sudden gains and losses in psychotherapy (Olthof, Hasselman, Strunk, et al., 2020). Several case studies also found support for the role of fluctuations in self-ratings as a precursor of depression onset (Wichers et al., 2020) and suicidal ideation (Fartacek et al., 2016). More studies at the within-person level are necessary to further examine the potential of early-warning signals for clinical change.

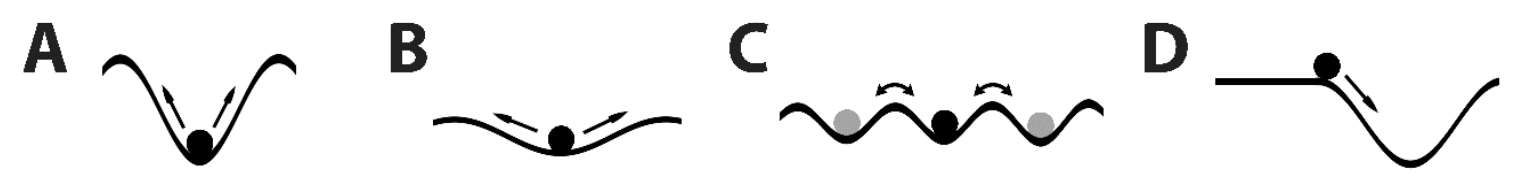

Figure 3. Conceptual illustration of a phase transition in a potential landscape. The depth of the valleys represent the attraction of accessible attractors whereas the ball indicates the current state of the system. Panel (A) shows a stable attractor: perturbations, indicated by the arrows will have minimal effects as the ball will immediately roll back into the valley. During a phase transition, such an initially stable attractor loses its stability (B), leading to increased (critical) fluctuations and an increased return time after perturbation (critical slowing down), until novel quasi-stable attractors emerge (C) that 'compete' for the attraction of the system until a tipping point (D) is reached.

\section{Control parameters}

If we conceptualize clinical change as a phase transition, it is crucial to identify control parameters that may cause such a change. Control parameters summarize the constraints that influence which attractors are available to the system, like leg weight and leg strength do for stepping behavior. In psychopathology, we propose that control parameters may be understood as akin to a stress-vulnerability framework (Hankin \& Abela, 2005). Stress can then be conceptualized as a collection of person-external constraints (stressors) and 


\section{COMPLEXITY THEORY OF PSYCHOPATHOLOGY}

vulnerability as a collection of person-internal constraints (all possible characteristics that make a person more or less vulnerable to stressors). Phase transitions in and out of psychopathology may then be driven by various idiographic processes that affect the global stress-vulnerability ratio in a multi-causal way (Sameroff, 2000).

Although the 'stress/vulnerability control parameter' provides a potentially fruitful way to think about causality in the development of psychopathology, we should be aware that stress and vulnerability can never be independent causes, as they are part of the interdependent person-environment system. A neat separation between cause and effect is therefore impossible, as the effects and causes directly feed back into each other ${ }^{3}$. Still, if the phenomenon of interest is a specific transition, it must in principle be possible to identify those processes that enabled a transition: a change in the stress/vulnerability ratio that functioned as 'the straw that broke the camel's back'. Such a causal effect, however, can only be determined by strict experimental manipulation of control parameters (Thelen \& Smith, 1994), which is usually not feasible in psychopathology research. Still, it may be possible to gain some insights in control parameters for clinical change.

Key for such an investigation is to study clinical change as it unfolds over time, as control parameters only become apparent close to a tipping point (Thelen \& Smith, 1994) ${ }^{4}$. Some case study results, for instance, suggest that tapering medication (Wichers et al., 2020) and increased feelings of restlessness (Smit et al., 2019) may function as control parameters in the onset of depression for some individuals. Of course, such observational studies cannot unambiguously identify control parameters, as other variables cannot be ruled out. Rather, they illustrate a research strategy - one of controlled observation over time, case by case -

\footnotetext{
${ }^{3}$ This is a central issue to all complex adaptive systems. For an excellent discussion of causality in complex systems see Van Geert (2019).

${ }^{4}$ This is even the case for well-understood phase transitions. For example, increasing temperature from 50 to 60 degrees of Celsius does not reveal heat as a causal influence on the transition between liquid water and gas: one must observe the system close to the critical point of 100 degrees of Celsius.
} 


\section{COMPLEXITY THEORY OF PSYCHOPATHOLOGY}

that may inspire applied clinical research to look into potential causal influences on clinical change for particular individuals in particular circumstances.

One last important aspect of control parameters for clinical change are nonlinear phenomena like hysteresis and enhanced contrast, which indicate that the accessibility of the attractors not only depends on a critical value of the control parameter, but also on the history of the system. Both phenomena refer to situations where a phase transition from state A to state B may occur at different values of the control parameter than a transition from state B to A.

Hysteresis concerns the phenomenon that systems often resist change. For example, an increase in stress at work may drive a transition into a depressed episode, but the elimination of stress at work (by quitting the job or taking sick leave), does not immediately reverse this transition (Cramer et al., 2016). Hysteresis thus means that transitions back into a previous attractor can lag behind changes in the control parameter. Enhanced contrast, in turn, concerns transitions that occur 'early'; that is, transitions are observed at values of the control parameter which are normally not associated with the onset of transitions. For example, the number of previously experienced depressed episodes due to stress at work may trigger the onset of an episode at increasingly lower levels of perceived stress. Phenomena like hysteresis and enhanced contrast underscore the importance of preventive efforts because preventing phase transitions is much easier than reversing them, as clearly illustrated in research on ecosystems (e.g. desertification is hard to reverse; Scheffer et al., 2009). Combined with the multi-causal nature of control parameters, hysteresis also explains why successful interventions are not necessarily aimed at 'reversing' the processes that caused psychopathology in the first place.

\section{Implications}




\section{COMPLEXITY THEORY OF PSYCHOPATHOLOGY}

The complexity theory of psychopathology we outline above has far-reaching implications for our conceptualization of psychopathology as well as for classification, intervention, and public health. The first of these is most pressing: what is psychopathology within this theory? We previously explained that both psychopathology and mental health can be conceptualized as dynamic patterns. Psychopathology is therefore not the disruption of a healthy pattern (e.g., a disbalance in humors, neurotransmitters, hormones, or personality traits; cf., Deyoung \& Krueger, 2018), but a pattern in itself. In other words, pathology is not a disorder, but another kind of order (Bosman, 2017).

Our theory does not imply an objective demarcation between psychopathology and mental health. Whilst dynamic patterns associated with psychopathology may have properties that can be recognized as problematic - such as being overly rigid - we believe that none of these properties can be identified as sufficient conditions for labeling patterns as 'psychopathological'. The property of being psychopathological should instead be understood as assigned to dynamic patterns that either others or the individuals themselves consider to be problematic in specific contexts (Bosman, 2017). What is considered psychopathology and what not is thus primarily dependent on societal or cultural norms and/or expectations (Bentall, 1993). Whilst such culturally informed judgments might coincide with the presence of more objective criteria such as maladaptivity, bio-markers or the aforementioned rigidity, they are not necessarily bound by these. There are always other cultural contexts imaginable in which such characteristics are considered healthy instead (Bosman, 2017). We agree with Canguilhem (1966) that suffering as experienced by the individual, or in extreme cases, those surrounding the individual, provides the only tenable criterion for psychopathology.

Second, following our conceptualization, there is no reason to expect that dynamic patterns evaluated as psychopathological allow for unambiguous classification in specific categories, such as those of the DSM-V, or any other classification system. Classification may 


\section{COMPLEXITY THEORY OF PSYCHOPATHOLOGY}

still be useful, but will never be optimal for clinical case formulation, as dynamic patterns of psychopathology are likely to be highly individualized. For case formulation, contextualprecision diagnosis may instead be helpful (van Os, et al., 2013), in which individual psychopathology is contextually examined through interviewing and process-monitoring with experience sampling techniques (e.g., Burger et al., 2020; Schiepek et al., 2016).

Third, our theory implies a different perspective on interventions. Interventions do not induce change in the patient following a form of 'billiard-ball causality', but interact with the individual patient over time (Hayes et al., 2007; Stiles \& Shapiro, 1994). Interventions then should be considered as a process aimed at reorganization and ideally adapt to the state the patient is in. For instance, if the psychopathological attractor is strong, treatment should aim at destabilization, but when the psychopathological attractor is destabilized, treatment should aim at supporting alternative, more healthy attractors (Hayes et al., 2015; Schiepek et al., 2016). This view on interventions fits historically well with ideas about stability and change in psychotherapy (Gelo \& Salvatore, 2016). Perhaps, the formalization of these ideas in terms of attractors and phase transitions provides a next step for intervention science in psychotherapy (e.g., Olthof et al., 2020), but also medical treatment (e.g., Wichers et al., 2020).

Fourth, understanding psychopathology as a dynamic pattern - rather than a (biologically) determined fixed condition - may reduce stigma and increase empowerment in patients. Importantly, both scientific and cultural beliefs about psychopathology affect how individuals see themselves (Hacking, 2007). The belief that change is possible, as psychopathology is not necessarily a fixed condition, can benefit feelings of empowerment (e.g., Lewis, 2018) and sometimes even lead to positive health effects (Schleider \& Weisz, 2018). The proposal by van Os (2016) to drop the term 'schizophrenia' (with the connotation of a 'hopeless chronic brain disease') and replace it by '(vulnerability to) psychosis' is a key 


\section{COMPLEXITY THEORY OF PSYCHOPATHOLOGY}

example of a shift towards a dynamic pattern conceptualization that potentially contributes to such goals. Nonetheless, we must realize that attractors in psychopathology may attract very strongly; not only in the sense that change may take longer for individuals with such attractors, but also that the attractor itself may be more or less permanent due to personinternal constraints limiting the system's degrees of freedom in a rather fixed manner. Genetic disorders, where a specific genetic effect dramatically constrains pattern formation, could for example be understood in such a way. Yet, whilst phase transitions from 'psychopathological' to 'normal' patterns may be difficult to achieve in these cases, significant quality of life improvements within the psychopathological pattern may be possible.

Fifth, our theory highlights the importance of the environment in conceptualizing psychopathology. Psychopathology emerges in a person-environment system and is thus not reducible to the person alone. Treatment therefore does not need not to be (solely) aimed at the person, but can also target the environment. For example, the relation between urban living and psychosis risk has inspired interventions in city planning such as creating green spaces, which have shown to reduce the incidence of psychosis (Baumann et al., 2020). The crucial role of the environment in psychopathology thus also points to a responsibility for institutions, such as schools, governments, and workplaces, that structure and influence the daily environment of individuals. Institutions can address mental health problems by improving circumstances that contribute to these problems, which in some cases will be more effective and cost-efficient than offering counseling or coaching to specific individuals.

To conclude, we advocate that a complexity theory of psychopathology will contribute to an emerging transdiagnostic science of psychopathology that combines research on personalized psychopathology with research on general principles of pattern formation. Such a science may bridge the gap between science and clinical practice, and support developments in case conceptualization, process-monitoring and personalized intervention which eventually 


\section{COMPLEXITY THEORY OF PSYCHOPATHOLOGY}

may benefit treatment efficacy. Last, we hope that a complex systems approach to psychopathology can contribute to a public health service and culture that de-stigmatizes psychopathology and empowers those who suffer from it.

\section{Acknowledgements}

We are grateful to Jerillyn Kent for her thorough and thoughtful comments during the editorial process, which have been extremely helpful for improving the final manuscript. We thank Nina de Boer, Olga de Bont, Anna Dapprich and Marieke Glazenburg for their feedback on previous versions of this manuscript. We thank Ralf Cox for producing Figure 1, Jingmeng Cui for co-producing Figure 2, and Nastasia Griffioen for producing Figure 3.

\section{Disclosure}

The authors report no conflict of interest.

\section{References}

Aderka, I. M., \& Shalom, J. G. (2021). A revised theory of sudden gains in psychological treatments. Behaviour Research and Therapy, 139, 103830.

Allsopp, K., Read, J., Corcoran, R., \& Kinderman, P. (2019). Heterogeneity in psychiatric diagnostic classification. Psychiatry Research, 279(June), 15-22. https://doi.org/10.1016/j.psychres.2019.07.005

Anderson, P. W. (1972). More is different. Science, 177(4047), 393-396. http://dx.doi.org/10.1126/science.177.4047.393

Baumann, P. S., Söderström, O., Abrahamyan Empson, L., Söderström, D., Codeluppi, Z., Golay, P., Birchwood, M., \& Conus, P. (2020). Urban remediation: a new recoveryoriented strategy to manage urban stress after first-episode psychosis. Social Psychiatry 


\section{COMPLEXITY THEORY OF PSYCHOPATHOLOGY}

and Psychiatric Epidemiology, 55(3), 273-283. https://doi.org/10.1007/s00127-01901795-7

Bentall, R. P. (1993). A proposal to classify happiness as a psychiatric disorder. British Journal of Psychiatry, 162(APR.), 539-542. https://doi.org/10.1192/bjp.162.4.539

Bernstein, N. (1967). The regulation and coordination of movements. Oxford: Pergamon press.

Bolbecker, A. R., Hong, S. L., Kent, J. S., Klaunig, M. J., O’Donnell, B. F., \& Hetrick, W. P. (2011). Postural control in bipolar disorder: Increased sway area and decreased dynamical complexity. PLoS ONE, 6(5). https://doi.org/10.1371/journal.pone.0019824

Bonnano, G. A., Papa, A., Lalande, K., Westphal, M., \& Coifman, K. (2005). The importance of being flexible. Psychological Science, 10, 482-487. https://doi.org/10.1038/nm.3341

Borsboom, D. (2016). A network theory of mental disorders. World Psychiatry, 16(1), 5-13. https://doi.org/10.1002/wps.20375

Borsboom, D., \& Cramer, A. O. J. (2013). Network analysis: An integrative approach to the structure of psychopathology. Annual Review of Clinical Psychology, 9(1), 91-121. https://doi.org/10.1146/annurev-clinpsy-050212-185608

Bos, E. H., \& De Jonge, P. (2014). "Critical slowing down in depression" is a great idea that still needs empirical proof. Proceedings of the National Academy of Sciences, 111(10), E878. https://doi.org/10.1073/pnas.1323672111

Bosman, A. M. T. (2017). Disorders are reduced normativity emerging from the relationship between organisms and their environment. In K. Hens, D. Cutas, \& D. Horstkötter (Eds.), Parental responsibility in the context of neuroscience and genetics (pp. 35-54). Springer International Publishing. https://doi.org/10.1007/978-3-319-42834-5_3

Bronfenbrenner, U. (1977). Toward an experimental ecology of human development. American Psychologist, 32(7), 513-531. https://doi.org/10.1037/0003-066X.32.7.513 


\section{COMPLEXITY THEORY OF PSYCHOPATHOLOGY}

Burger, J., van der Veen, D. C., Robinaugh, D., Quax, R., Riese, H., Schoevers, R. A., \& Epskamp, S. (2020). Bridging the gap between complexity science and clinical practice by formalizing idiographic theories: A computational model of functional analysis. BMC Medicine, 18. https://doi.org/10.1186/s12916-020-01558-1

Canguilhem, G. (1966). The normal and the pathological. Zone Books.

Caspi, A., Houts, R. M., Ambler, A., Danese, A., Elliott, M. L., Hariri, A., Harrington, H. L., Hogan, S., Poulton, R., Ramrakha, S., Rasmussen, L. J. H., Reuben, A., RichmondRakerd, L., Sugden, K., Wertz, J., Williams, B. S., \& Moffitt, T. E. (2020). Longitudinal assessment of mental health disorders and comorbidities across 4 decades among participants in the dunedin birth cohort study. JAMA Network Open, 3(4), e203221. https://doi.org/10.1001/jamanetworkopen.2020.3221

Cicchetti, D., \& Cohen, D. J. (Eds.). (2006). Developmental psychopathology: Theory and method (2nd ed.). John Wiley \& Sons, Inc.

Colombetti, G. (2012). Psychopathology and the enactive mind. In Oxford Handbook of Philosophy of Psychiatry (pp. 1-12). Oxford University Press. https://doi.org/10.1093/oxfordhb/9780199579563.013.0063

Cramer, A. O., Van Borkulo, C. D., Giltay, E. J., Van Der Maas, H. L., Kendler, K. S., Scheffer, M., \& Borsboom, D. (2016). Major depression as a complex dynamic system. PloS one, 11(12), e0167490. https://doi.org/10.1371/journal.pone.016749

de Haan, S. (2020). An enactive approach to psychiatry. Philosophy, Psychiatry and Psychology, 27(1), 3-25. https://doi.org/10.1353/ppp.2020.0001

DeYoung, C. G., \& Krueger, R. F. (2018). A cybernetic theory of psychopathology. Psychological Inquiry, 29(3), 117-138. https://doi.org/10.1080/1047840X.2018.1513680

Engel, G. L. (1977). The need for a new medical model: A challenge for biomedicine. Science, 196, 129-135. https://doi.org/10.1097/smj.0b013e318208767b 


\section{COMPLEXITY THEORY OF PSYCHOPATHOLOGY}

Eronen, M. I. (2019). The levels problem in psychopathology. Psychological Medicine, 1-7. https://doi.org/10.1017/S0033291719002514

Fartacek, C., Schiepek, G., Kunrath, S., Fartacek, R., \& Plöderl, M. (2016). Real-Time Monitoring of non-linear suicidal dynamics: Methodology and a demonstrative case report. Frontiers in Psychology, 7(2), 1-14. https://doi.org/10.3389/fpsyg.2016.00130

Fausto-sterling, A. (2020). The bare bones of sex: Part1-sex and gender. Women, Science, and Technology, 30(2), 233-250. https://doi.org/10.4324/9780203895658-25

Fisher, A. J., \& Newman, M. G. (2016). Reductions in the diurnal rigidity of anxiety predict treatment outcome in cognitive behavioral therapy for generalized anxiety disorder. Behaviour Research and Therapy, 79, 46-55. https://doi.org/10.1016/j.brat.2016.02.006

Freeman, W. J. (1992). Tutorial on neurobiology: From single neurons to brain chaos. International Journal of Bifurcation and Chaos, 2, 451-482. https://doi.org/10.1142/S0218127492000653

Frisch, S. (2016). Are mental disorders brain diseases, and what does this mean? A clinicalneuropsychological perspective. Psychopathology, 49(3), 135-142. https://doi.org/10.1159/000447359

Gelo, O. C. G., \& Salvatore, S. (2016). A dynamic systems approach to psychotherapy: A meta-theoretical framework for explaining psychotherapy change processes. Journal of Counseling Psychology, 63, 379-395. https://doi.org/10.1037/cou0000150

Gilden, D. L., \& Hancock, H. (2007). Response variability in attention-deficit disorders. Psychological Science, 18(9), 796-802. https://doi.org/10.1111/j.14679280.2007.01982.x

Goldberger, A. L., Amaral, L. A. N., Hausdorff, J. M., Ivanov, P. C., Peng, C. K., \& Stanley, H. E. (2002). Fractal dynamics in physiology: Alterations with disease and aging. Proceedings of the National Academy of Sciences of the United States of America, 


\section{COMPLEXITY THEORY OF PSYCHOPATHOLOGY}

99(SUPPL. 1), 2466-2472. https://doi.org/10.1073/pnas.012579499

Granic, I. (2005). Timing is everything: Developmental psychopathology from a dynamic systems perspective. Developmental Review, 25(3-4), 386-407.

https://doi.org/10.1016/j.dr.2005.10.005

Hacking, I. (2007). Kinds of people: Moving targets. Proceedings of the British Academy, Volume 151, 2006 Lectures, 285-317.

https://doi.org/10.5871/bacad/9780197264249.003.0010

Haken, H. (1983). Synergetics: an introduction. Non-equilibrium phase transition and selfselforganisation in physics, chemistry and biology. Springer Verlag.

Haken, H. (1992). Synergetics in Psychology. 32-54. https://doi.org/10.1007/978-3-642$77534-5 \_2$

Hankin, B. L., \& Abela, J. R. Z. (2005). Development of psychopathology: A vulnerabilitystress perspective. Development of Psychopathology: A Vulnerability-Stress Perspective, 1-510. https://doi.org/10.4135/9781452231655

Haslbeck, J., Ryan, O., Robinaugh, D., Waldorp, L., \& Borsboom, D. (2019). Modeling Psychopathology: From Data Models to Formal Theories. PsyArxiv. https://doi.org/10.31234/osf.io/jgm7f

Hasselman, F. (2015). Beyond the Boundary An analysis of verisimilitude and causal ontology of scientific claims Atiologies of developmental dyslexia as a case in point. [Doctoral dissertation, Radboud University]. Radboud Repository. http://hdl.handle.net/2066/140654

Hayes, A. M., \& Andrews, L. A. (2020). A complex systems approach to the study of change in psychotherapy. BMC Medicine, 18(1), 1-13. https://doi.org/10.1186/s12916-02001662-2

Hayes, A. M., Laurenceau, J.-P., Feldman, G., Strauss, J. L., \& Cardaciotto, L. (2007). 


\section{COMPLEXITY THEORY OF PSYCHOPATHOLOGY}

Change is not always linear: the study of nonlinear and discontinuous patterns of change in psychotherapy. Clinical Psychology Review, 27, 715-723.

https://doi.org/10.1016/j.cpr.2007.01.008.Change

Hayes, A. M., Yasinski, C., Ben Barnes, J., \& Bockting, C. L. H. (2015). Network destabilization and transition in depression: New methods for studying the dynamics of therapeutic change. Clinical Psychology Review, 41, 27-39.

https://doi.org/10.1016/j.cpr.2015.06.007

Hollenstein, T., Lichtwarck-Aschoff, A., \& Potworowski, G. (2013). A model of socioemotional flexibility at three time scales. Emotion Review, 5(4), 397-405. https://doi.org/10.1177/1754073913484181

Holtzheimer, P. E., \& Mayberg, H. S. (2011). Stuck in a rut: Rethinking depression and its treatment. Trends in Neurosciences, 34(1), 1-9. https://doi.org/10.1016/j.tins.2010.10.004

Kéfi, S., Dakos, V., Scheffer, M., Van Nes, E. H., \& Rietkerk, M. (2013). Early warning signals also precede non-catastrophic transitions. Oikos, 122(5), 641-648. https://doi.org/10.1111/j.1600-0706.2012.20838.x

Kelso, J. A. S. (1997). Dynamic patterns: The self-organization of brain and behavior. MIT press.

Kelso, J. A. S. (2012). Multistability and metastability: understanding dynamic coordination in the brain. Philosophical Transactions of the Royal Society B: Biological Sciences, 367(1591), 906-918. https://doi.org/10.1098/rstb.2011.0351

Kelso, J. A. S., Scholz, J. P., \& Schöner, G. (1986). Nonequilibrium phase transitions in coordinated biological motion: critical fluctuations. Physics Letters A, 118(6), 279-284. https://doi.org/10.1016/0375-9601(86)90359-2

Kendler, K. S., Zachar, P., \& Craver, C. (2011). What kinds of things are psychiatric 


\section{COMPLEXITY THEORY OF PSYCHOPATHOLOGY}

disorders? Psychological Medicine, 41(6), 1143-1150.

https://doi.org/10.1017/S0033291710001844

Kent, J. S., Hong, S. L., Bolbecker, A. R., Klaunig, M. J., Forsyth, J. K., O’Donnell, B. F., \& Hetrick, W. P. (2012). Motor deficits in schizophrenia quantified by nonlinear analysis of postural sway. PLoS ONE, 7(8). https://doi.org/10.1371/journal.pone.0041808

Kessler, R. C., Chiu, W. T., Demler, O., \& Walters, E. E. (2005). Prevalence, severity, and comorbidity of 12-month DSM-IV disorders in the national comorbidity survey replication. Archives of General Psychiatry, 62(6), 617-627.

https://doi.org/10.1001/archpsyc.62.6.617

Ladyman, J., Lambert, J., \& Wiesner, K. (2013). What is a complex system? European Journal for Philosophy of Science, 3(1), 33-67. https://doi.org/10.1007/s13194-0120056-8

Lewis, M. (2018). Brain change in addiction as learning, not disease. New England Journal of Medicine, 379(16), 1551-1560. https://doi.org/10.1056/NEJMc1815144.

Lutz, W., Ehrlich, T., Rubel, J., Hallwachs, N., Röttger, M. A., Jorasz, C., Mocanu, S., Vocks, S., Schulte, D., \& Tschitsaz-Stucki, A. (2013). The ups and downs of psychotherapy: Sudden gains and sudden losses identified with session reports. Psychotherapy Research, 23(1), 14-24. https://doi.org/10.1080/10503307.2012.693837

Mahoney, M. J. (1991). Human change processes: The scientific foundations of psychotherapy. BasicBooks.

Masten, A. S. (2001). Ordinary magic: Resilience processes in development. American Psychologist, 56(3), 227. http://dx.doi.org/10.1037/0003-066X.56.3.227

Nelson, B., McGorry, P. D., Wichers, M., Wigman, J. T. W., \& Hartmann, J. A. (2017). Moving from static to dynamic models of the onset of mental disorder. JAMA Psychiatry, 74, 528-534. https://doi.org/10.1001/jamapsychiatry.2017.0001 


\section{COMPLEXITY THEORY OF PSYCHOPATHOLOGY}

Olthof, M., Hasselman, F., \& Lichtwarck-Aschoff, A. (2020). Complexity in psychological self-ratings: Implications for research and practice. BMC Medicine, 18(1), 1-16. https://doi.org/10.1186/s12916-020-01727-2

Olthof, M., Hasselman, F., Strunk, G., van Rooij, M., Aas, B., Helmich, M. A., Schiepek, G., \& Lichtwarck-Aschoff, A. (2020). Critical fluctuations as an early-warning signal for sudden gains and losses in patients receiving psychotherapy for mood disorders. Clinical Psychological Science, 8(1), 25-35. https://doi.org/10.1177/2167702619865969

Richters, J. E. (1997). The Hubble hypothesis and the developmentalist's dilemma. Development and Psychopathology, 9(2), 193-229. https://doi.org/10.1017/s0954579497002022

Robinaugh, D., Haslbeck, J., Waldorp, L., Kossakowski, J., Fried, E. I., Millner, A., McNally, R. J., van Nes, E. H., Scheffer, M., \& Kendler, K. S. (2019). Advancing the network theory of mental disorders: A computational model of panic disorder. Psyarxiv. https://doi.org/10.31234/osf.io/km37w

Sameroff, A. J. (2000). Developmental systems and psychopathology. Development and Psychopathology, 12(3), 297-312. https://doi.org/10.1017/S0954579400003035

Scheffer, M., Bascompte, J., Brock, W. A., Brovkin, V., Carpenter, S. R., Dakos, V., Held, H., van Nes, E. H., Rietkerk, M., \& Sugihara, G. (2009). Early-warning signals for critical transitions. Nature, 461(7260), 53-59. https://doi.org/10.1038/nature08227 Schiepek, G. (2003). A dynamic systems approach to clinical case formulation. European Journal of Psychological Assessment, 19, 175-184. https://doi.org/10.1027//10155759.19 .3 .175

Schiepek, G., Eckert, H., Aas, B., Wallot, S., \& Wallot, A. (2016). Integrative psychotherapy: A feedback-driven dynamic systems approach. Hogrefe. https://doi.org/10.1027/00472000 


\section{COMPLEXITY THEORY OF PSYCHOPATHOLOGY}

Schiepek, G., \& Tschacher, W. (1992). Application of synergetics to clinical psychology. In W. Tschacher, G. Schiepek, \& E. J. Brunner (Eds.), Self-Organization and Clinical Psychology (pp. 3-31). Springer

Schleider, J., \& Weisz, J. (2018). A single-session growth mindset intervention for adolescent anxiety and depression: 9-month outcomes of a randomized trial. Journal of Child Psychology and Psychiatry, 59, 160-170. https://doi.org/10.1111/jcpp.12811

Schöner, G., Jiang, W. Y., \& Kelso, J. A. S. (1990). A synergetic theory of quadrupedal gaits and gait transitions. Journal of Theoretical Biology, 142, 359-391. https://doi.org/10.1016/S0022-5193(05)80558-2

Schöner, G., \& Kelso, J. A. (1988). Dynamic pattern generation in behavioral and neural systems. Science, 239(4847), 1513-1520. https://doi.org/10.1126/science.3281253

Shalom, J. G., \& Aderka, I. M. (2020). A meta-analysis of sudden gains in psychotherapy: Outcome and moderators. Clinical Psychology Review, 76(January), 101827. https://doi.org/10.1016/j.cpr.2020.101827

Smit, A. C., Snippe, E., \& Wichers, M. (2019). Increasing restlessness signals impending increase in depressive symptoms more than 2 months before it happens in individual patients. Psychotherapy and Psychosomatics, 88(4), 249-251. https://doi.org/10.1159/000500594

Stiles, W. B., \& Shapiro, D. A. (1994). Disabuse of the drug metaphor: Psychotherapy process-outcome correlations. Journal of Consulting and Clinical Psychology, 62(5), 942-948. https://doi.org/10.1037/0022-006X.62.5.942

Strogatz, S. H. (2015). Nonlinear dynamics and chaos. CRC Press. https://doi.org/10.1201/9780429492563

Takens F. (1981) Detecting strange attractors in turbulence. In D. Rand, \& L. S. Young (Eds.), Dynamical systems and turbulence (pp. 366-381). Springer. 


\section{COMPLEXITY THEORY OF PSYCHOPATHOLOGY}

https://doi.org/10.1007/BFb0091924

Tang, T. Z. ., \& DeRubeis, R. J. (1999). Sudden gains and critical sessions in cognitivebehavioral therapy for depression. Journal of Consulting and Clinical Psychology, 6, 894-904. http://dx.doi.org/10.1037/0022-006X.67.6.894

Thelen, E., \& Smith, L. B. (1994). A dynamic systems approach to the development of cognition and action. The MIT Press.

Thelen, E., \& Ullrich, B. D. (1991). Hidden skills: A dynamic systems analysis of treadmill stepping during the first year. Monographs of the Society for Research in Child Development, 56(1), 1-103. http://dx.doi.org/10.2307/1166099

Turvey, M. T. (1990). Coordination. American Psychologist, 45(8), 938-953. https://doi.org/10.1007/s00422-003-0460-4

Turvey, M. T. (2007). Action and perception at the level of synergies. Human Movement Science, 26(4), 657-697. https://doi.org/10.1016/j.humov.2007.04.002

van de Leemput, I. A., Wichers, M., Cramer, A. O. J., Borsboom, D., Tuerlinckx, F., Kuppens, P., van Nes, E. H., Viechtbauer, W., Giltay, E. J., Aggen, S. H., Derom, C., Jacobs, N., Kendler, K. S., van der Maas, H. L. J., Neale, M. C., Peeters, F., Thiery, E., Zachar, P., \& Scheffer, M. (2014). Critical slowing down as early warning for the onset and termination of depression. Proceedings of the National Academy of Sciences, 111, 87-92. https://doi.org/10.1073/pnas.1312114110

Van Geert, P. L. C. (2019). Dynamic systems, process and development. Human Development, 63(3-4), 153-179. https://doi.org/10.1159/000503825

Van Orden, G. C., Holden, J. G., \& Turvey, M. T. (2003). Self-organization of cognitive performance. Journal of Experimental Psychology: General, 132(3), 331-350. https://doi.org/10.1037/0096-3445.132.3.331

Van Orden, G. C., Kloos, H., \& Wallot, S. (2011). Living in the pink. Intentionality, 


\section{COMPLEXITY THEORY OF PSYCHOPATHOLOGY}

wellbeing, and complexity. In C. Hooker (Ed.), Handbook of the philosophy of Science. Vol 10. Philosophy of Complex Systems (pp. 639-683). Elsevier. https://doi.org/10.1016/B978-0-444-52076-0.50022-5

van Os, J. (2016). “Schizophrenia” does not exist. British Medical Journal, 352, i375. https://doi.org/10.1136/bmj.i375

van Os, J., Guloksuz, S., Vijn, T. W., Hafkenscheid, A., \& Delespaul, P. (2019). The evidence-based group-level symptom-reduction model as the organizing principle for mental health care: time for change? World Psychiatry, 18(1), 88-96. https://doi.org/10.1002/wps.20609

Vittengl, J. R., Clark, L. A., \& Jarrett, R. B. (2005). Validity of sudden gains in acute phase treatment of depression. Journal of Consulting and Clinical Psychology, 73(1), 173-182. https://doi.org/10.1037/0022-006X.73.1.173

Wallot, S., \& Kelty-Stephen, D. G. (2017). Interaction-dominant causation in mind and brain, and its implication for questions of generalization and replication. Minds and Machines, 28, 353-374. https://doi.org/10.1007/s11023-017-9455-0

Wichers, M., Smit, A. C., \& Snippe, E. (2020). Early warning signals based on momentary affect dynamics can expose nearby transitions in depression: A confirmatory singlesubject time-series study. Journal for Person-Oriented Research, 6(1), 1-15. https://doi.org/10.17505/jpor.2020.22042

Wijnants, M. L. (2014). A review of theoretical perspectives in cognitive science on the presence of 1 /f scaling in coordinated physiological and cognitive processes. Journal of Nonlinear Dynamics, Volume 2014, Article 962043.

https://doi.org/10.1155/2014/962043

Wijnants, M. L., Hasselman, F., Cox, R. F. A., Bosman, A. M. T., \& van Orden, G. (2012). An interaction-dominant perspective on reading fluency and dyslexia. Annals of 


\section{COMPLEXITY THEORY OF PSYCHOPATHOLOGY}

Dyslexia, 62(2), 100-119. https://doi.org/10.1007/s11881-012-0067-3

Wittenborn, A. K., Rahmandad, H., Rick, J., \& Hosseinichimeh, N. (2016). Depression as a systemic syndrome: Mapping the feedback loops of major depressive disorder. Psychological Medicine, 46(3), 551-562. https://doi.org/10.1017/S0033291715002044

Wright, A. G. C., \& Woods, W. C. (2020). Personalized models of psychopathology. Annual Review of Clinical Psychology, 16, 49-74. https://doi.org/10.1146/annurev-clinpsy$102419-125032$

Zilcha-Mano, S., Errázuriz, P., \& Derubeis, R. J. (2019). Are there any robust predictors of "sudden gainers," and how is sustained improvement in treatment outcome achieved following a gain? Journal of Consulting and Clinical Psychology, 87(6), 491-500. https://doi.org/10.1037/ccp0000401.supp 